\title{
The Hippocampal Cognitive Map: One Space or Many?
}

\author{
Hugo. J. Spiers
}

Institute of Behavioural Neuroscience, Department of Experimental Psychology, Division of Psychology and Language Sciences, University College London

26 Bedford Way, WC1H OAP, UK

Email contact: h.spiers@ucl.ac.uk

\begin{abstract}
New evidence that hippocampal activity tracks distance in semantic space during recall (Solomon et al., PNAS) supports a growing consensus of a domain-general cognitive map. But are all inputs equally processed into a 'universal map' or are there input constraints (e.g. space, semantics) leading to differentiated multiple maps across the hippocampus with distinct properties?
\end{abstract}

\section{Main body text:}

Observing the impressive learning of rats in complex mazes, Edward Tolman concluded their brain must form an internal representation of the environment that transcended individual associations between maze-junctions and actions needed for reward [1]. He termed this system a 'cognitive map' and argued humans share this with rodents. He also postulated that humans map abstract entities. The discovery of 'place cells' in the rodent hippocampus, which code for location in the environment via their spatially localised firing patterns, led O'Keefe and Nadel [2] to propose that the hippocampus was the neural locus of the cognitive map, endowing an animal with an environment-centric spatial memory system which, in a literal sense, mapped space. The hallmark of this hippocampal map was the capacity to take efficient detours and exploit shortcuts through unexplored space. The decades that followed the discovery of place cells witnessed an explosion in the discovery of spatial coding cells within and beyond the hippocampus and their extension to humans [3].

Recently researchers have revisited Tolman's original suggestion that the hippocampus also maps non-spatial information [3,4]. The argument that space is simply one of many entities processed by the hippocampus has long been argued [5], but recent evidence reveals that non-spatial information can be 'mapped' in the hippocampus along stimulus dimensions, such as sound frequencies, visual properties or social hierarchies [3, 4]. Notably, O'Keefe and Nadel [2] also argued that the human hippocampus would map abstract space due the encroachment of language into the temporal lobes but did not anticipate the full breadth of this mapping. This 'universal cognitive map' perspective has gained greater traction with the proposal that entorhinal cortical inputs to the hippocampus provide a compressed representation of the transition structure in the environment, both for spatial and nonspatial elements [4].

\section{New Evidence from Semantic Spaces}

Much of the recent evidence for non-spatial mapping has come from indirect measures of neural activity using neuroimaging and the use of tasks abstracted from everyday experience. In a new study, Solomon and colleagues [6] explored the everyday experience of recalling a memorized word list and 
recorded direct neural activity - local field potential oscillations - in the hippocampus of neurosurgical patients. In such a task no overt mapping is required, just the capacity to memorise and recall words. Nonetheless, the authors were able to reveal that the power of hippocampal oscillations in the thetaband $(4-8 \mathrm{~Hz})$ just prior to recall of each word was correlated with the proximity in the semantic distance between words recalled. For example, theta oscillations would be large when recalling the word "Dog" after just recalling "Fur" (small sematic distance between Dog and Fur), but much smaller oscillations would occur when "Dog" was recalled just after "Pin" (large semantic distance between Dog and Pin). Semantic distance was defined using a word2vec model [6], which uses a vast corpus of words to determine the semantic relatedness of words along multiple extracted dimensions.

Temporal distance coding was also observed. If a word was recalled just after recalling a word close in the word list (e.g. recalling words 1 and 2 form a list of 7 words) then larger theta oscillations were evoked prior to recall. Both this temporal distance and the semantic distance are related to the ease of recall [6]. Because theta is a prominent oscillation linked to spatial navigation and distance coding $[2,7]$ these patterns can be interpreted as evidence that the hippocampus not only acts to retrieve information in a map of physical space, but that it also supports 'movement' along non-spatial dimensions during recall, in this case temporal and semantic distance.

\section{One Universal Map or Multiple Maps?}

The results are exciting because they show just how abstract the hippocampal code can be. The findings would appear to agree with a universal mapping system in the hippocampus that codes information of any kind, with physical space equivalent to abstract spaces [4]. However, an alternative possibility is that the hippocampus creates differently formed maps with distinct properties depending on what is being mapped.

It has been argued that space is special, and that the capacity to code abstract stimuli evolved from a foundational core spatial system [8]. Unlike the properties of objects and stimuli used in most nonspatial experiments, space cannot simply be removed from experience, it is fundamental to it [8]. The entry of spatial and non-spatial information into the hippocampus differs anatomically, with spatial information targeting more densely the posterior/dorsal regions and non-spatial information targeting anterior/ventral regions [9]. In humans, there is also a right hemisphere bias for spatial processing and a left hemisphere bias for verbal processing $[3,9,10]$. Thus, it seems logical that the tracking of distance in semantic space and physical space may well occur within different sub-regions of the hippocampus - thus forming differently distributed maps. Consistent with this, semantic distance related oscillations occurred most consistently in the left hemisphere [6], whereas tracking physical distance has been associated with the right posterior hippocampus [10].

If the hallmark of a cognitive map is the ability to take shortcuts and detours, then maps of abstract knowledge should also support this. However, currently there is little direct evidence for this. In the case of semantic spaces, these were coded by 1-D spaces [6], within which it is impossible to take a shortcut or a detour. It is possible to envisage efficient detour taking in a network such as a social hierarchy; if two people refuse to communicate, then a new detour route via 'go-betweens' could be inferred from the stored map of the social network. However, the difference between a social network and a physical network of paths is that paths are embedded in Euclidean space. This makes it possible to infer both novel topological connections and also non-topological vectors to find novel shortcuts through unexplored space. Thus, while the hippocampal circuit may have evolved the capacity to map diverse information [4], the maps it forms, and the anatomical locus of those maps, may differ depending on what is being mapped. How such maps may be integrated for solving problems across domains remains an exciting question for future research. 


\section{Acknowledgements}

HJS received support from the Alzheimer's Research UK and the Human Brain Project.

\section{References}

1. E.C. Tolman. Cognitive maps in rats and men Psych. Rev., 55 (1948), pp. 189-208

2. J. O'Keefe, L. Nadel. The hippocampus as a cognitive map Oxford: Clarendon Press (1978)

3. R. A. Epstein, et al. The cognitive map in humans: spatial navigation and beyond Nat. Neurosci., 20 (2017), pp. 1504-1513

4. T.E.J. Behrens, et al. What is a cognitive map? Organizing knowledge for flexible behaviour Neuron 100 (2018), pp. 490-509

5. H. Eichenbaum, N. J. Cohen Can we reconcile the declarative memory and spatial navigation views on hippocampal function? Neuron 83 (2014), pp. 764-770

6. EA Solomon, et al. Hippocampal theta codes for distances in semantic and temporal spaces Proc. Natl. Acad. Sci. U S A. 116 (2019), pp. 24343-24352

7. L. K. Vass, et al. Oscillations go the distance: Low-frequency human hippocampal oscillations code spatial distance in the absence of sensory cues during teleportation. Neuron 89 (2016), pp. 1180-1186

8. L. Nadel. The hippocampus and space revisited Hippocampus 1 (1991), pp. 221-229.

9. B.A. Strange et al. Functional organization of the hippocampal longitudinal axis Nat. Rev. Neurosci. 15 (2014), pp. 655-669

10. E.Z. Patai et al. Hippocampal and retrosplenial goal distance coding after long-term consolidation of a real-world environment Cereb. Cortex 29 (2019), pp. 2748-2758. 